\title{
INTEGRATING INTELLIGENCE IN HYDROPONIC FARMS
}

\author{
Dr. V.R. Saraswathy \\ Professor, Department of Electronics and Communication Engineering, Kongu Engineering \\ College, Erode, Tamilnadu, India \\ C. Nithiesh, S. Palani Kumaravel, S. Ruphasri \\ Department of Electronics and Communication Engineering, Kongu Engineering College, \\ Erode, Tamilnadu, India
}

\begin{abstract}
Agriculture plays a major role on improving the economy of the country. Modern farming technique like Hydroponics is in rise which is soilless farming culture and the plants were grown with the help of nutrient solutions. The Hydroponic system can be controlled by applying machine learning algorithms like Deep Neural Networks. Internet of Things (IoT) allows Machine to Machine communication and controlling the hydroponic system autonomously and intelligently. This work proposes to develop an intelligent IoT based hydroponic system by employing Deep Neural Networks. The system so developed is intelligent enough in providing the appropriate control over the sprinkler and water flow for the hydroponic environment based on the multiple input parameters gathered from sensors like $\mathrm{pH}$, temperature, humidity and water flow.
\end{abstract}

Key words: IoT, Agriculture, Neural Network, Intelligence, machine learning

Cite this Article: Dr. V.R. Saraswathy, C. Nithiesh, S. Palani Kumaravel, S. Ruphasri, Integrating Intelligence in Hydroponic Farms, International Journal of Electrical Engineering and Technology, 11(4), 2020, pp. 150-158.

https://iaeme.com/Home/issue/IJEET?Volume $=11 \&$ Issue $=4$

\section{INTRODUCTION}

With the advent of civilization, open field/soil-based agriculture is facing major challenges and most importantly decrease in land availability. Human population is increasing rapidly. Food production has to be increased to satisfy the increased population. Besides, less rainfall in some of the cultivable areas like Palladam, outer regions of Udumalpet, Thiruvarur etc., frequent drought conditions, unpredictability of climate and weather patterns, rise in temperature, river pollution, wastage of water, decline in ground water level, etc. are threatening factors when traditional farming technique is used. So it is proposed to go for less soil culture calledHydroponics. Hydroponics is a subset of hydro culture and is a method of growing plants using mineral nutrient solutions, in water, very little amount of soil. This 
farming generally requires very less water and less cultivable lands. Through Hydroponics, terrace farming is also possible which yields fresh crops (1).

Intelligence in the field of hydroponics make the system work automatically. This increases the productivity in agriculture with minimum water resources and available cultivable land. Automation is done through IoT which is Machine-to-Machine interaction. Recurrent Neural Network (RNN) -Long Short-Term Memory (LSTM) prediction algorithm would increase the accuracy in automation (2).

\section{LITERATURE REVIEW}

Research works for implementing Intelligent IoT Based hydroponics system by employing IoT and machine learning technologies are discussed here.

\subsection{IoT in Hydroponics}

IoT refers to the Internet of things towards connecting people, things by means of the Internet and store the data in the cloud for analysis. The emergence of IoT has allowed farmers to automate the hydroponic culture. Monitoring water level, $\mathrm{pH}$, temperature and light intensity can be done and they can be regulated by the use of IoT (3).

Shreya Tembe(2015) (4), have developed a IoT based Hydroponics system where the sensors are interfaced with Wi-Fi module for monitoring and the automated part includes sprinklers for pest, humidity adjustment unit and $\mathrm{pH}$ up/down pump accordingly

S.Keerthana(2018) (5), has developed an environment driven control methods which include sensors, LED lighting, water spray and water pump which can effectively lower the $\mathrm{CO}_{2}$ concentration, the temperature and increases the water level.

\subsection{Machine Learning in Hydroponics}

Machine learning is a subset of Artificial Intelligence (AI) that helps in providing computers capability to perform actions on its own after being trained for a particular task. Foremost, for a machine to think like a human mind, it has first to think and learn like a human being. Human mind 0 from the past experiences and past data that is exposed to and based on that the human being takes decision for the future. The machine learning algorithm has various uses in the field of hydroponics.

K. P. Ferentinos (6), has developed a deep neural network model with parameters of farming as input which would predict the conductivity and $\mathrm{pH}$ of the nutrient solutions.

From the literature survey, the drawbacks in the earlier system are that the automation developed employing IoT had no intelligence in producing the appropriate control action for the hydroponic environment based on parameters trained. In terms of machine level intelligence developed in hydroponics, they were not developed for IoT based system. The system here only concentrates on applying machine learning algorithms like Bayesian and ANN for predicting the appropriate control action based on parameters trained (7). The work did not focus on controlling the hydroponic environment.

So accordingly, this work is focused on developing an intelligent IoT based hydroponics where the control actions are based on real-time parameters gathered from the sensors of the hydroponics system. This control action is done through Raspberry-pi processed to work on prediction algorithm and controller. 


\section{PROPOSED METHOD}

The parameters like moisture, $\mathrm{pH}$, temperature, light intensity, water flow are collected by using the appropriate sensors from traditional farming and the values are uploaded to the cloud using Node MCU.

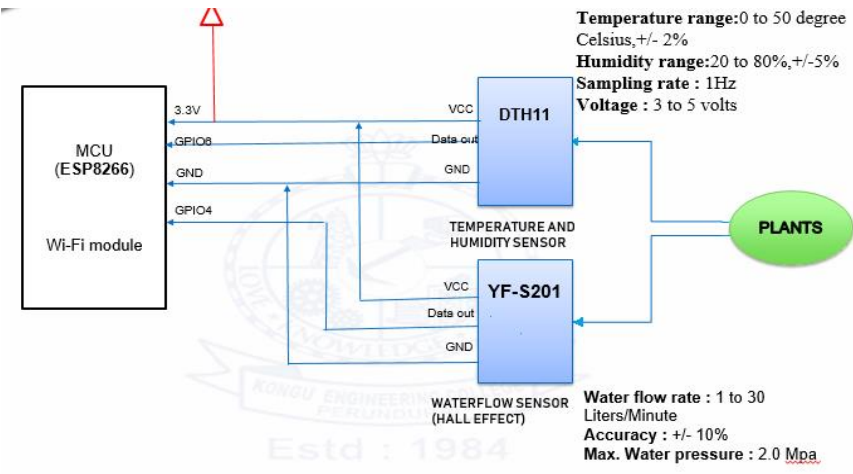

Figure 1 Circuit for collecting data from traditional farming

Hydroponics system based on Nutrient film technique (NFT) is implemented where the water with nutrients continuously flowing over the roots, this ensures that bottom part of the root gets proper humidity and the upper part gets more oxygen and it remains dry.

\subsection{IoT based Hydroponics System}

The parameters from Hydroponics system are also collected by using the appropriate sensors. Existing systems in hydroponics needs a control action based on the parameters gathered (8). In this work, the control action towards the Hydroponics system is done through controller.

This work has an Intelligent IoT based hydroponics system by employing LSTM based RNN for better accuracy compared with other networks. Here, the system captures the parameters like $\mathrm{pH}$, Temperature, light intensity, humidity, Water level which are monitored and accordingly analyzed by applying RNN-LSTM Network for predicting the appropriate control action towards controlling the hydroponic system.

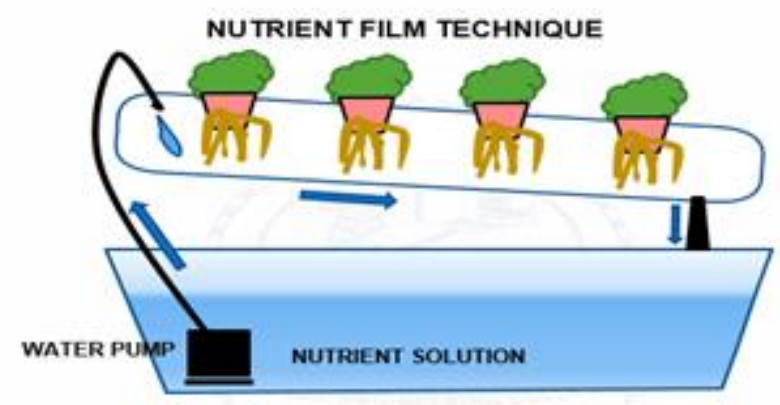

Figure 2 Hydroponics setup using NFT technique 


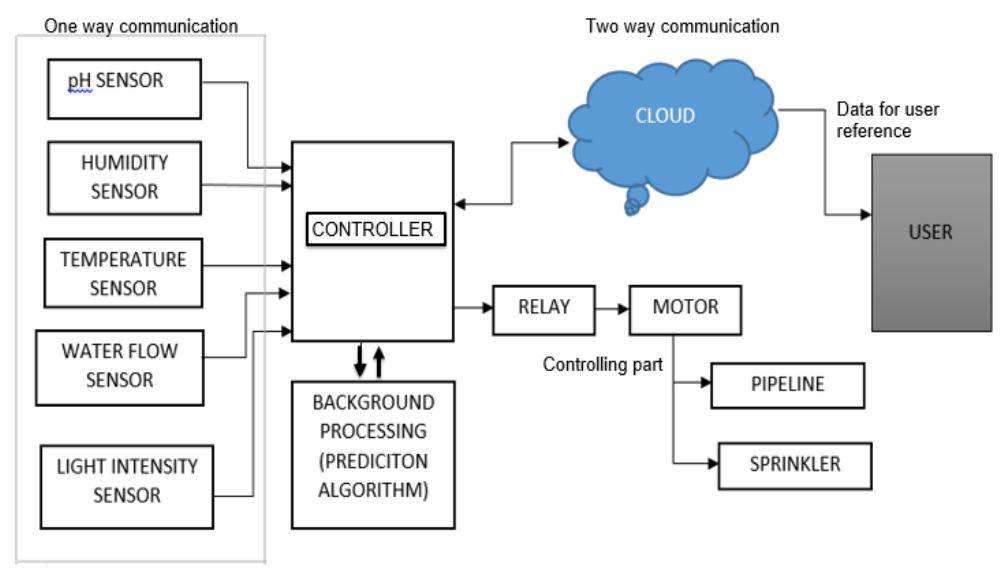

Figure 3 Block diagram for implementing IoT based Hydroponics

\subsection{Recurrent Neural Network (RNN)}

Multi layered perceptron (MLP) maps only from input to output vectors. RNN can map from the entire history of previous inputs to each output. RNNS' are designed to work with sequence predictions. RNNs have internal cell state memory to process the sequence of inputs. RNN model predicts the value of humidity, temperature, water level. By using obtained value from the algorithm the system is controlled using IoT which would make the Hydroponics system to be more accurate.

The data gathered from the sensors are given as input to the RNN and it produces the output which can be either normal or abnormal. RNNs have neuron-like nodes organized into successive layer. Each connection has a modifiable real-valued weight. Each sequence in RNN produces an error of deviation in values.

RNN uses Back-Propagation through time to learn, which is made possible using activation function.Back propagation is an algorithm which traces back from the output of the model, suggests an optimal weight for each neuron which results in the most accurate prediction. Each node in RNN contains an internal cell state called hidden state. The RNN consists of fixed activation function units, one for each time step.Activation Function helps to find whether it should be activated or not, based on whether the input given to each neuron is appropriate for the prediction of data. Activation functions also helps to round-off the each neuron's output to a range of 1 and 0 or -1 and 1 . RNN models use non-linear activation functions. The non-linear activation function allows back propagation because they have a derivative function which is related to the inputs. Sigmoid and tanh are used in this work (9).

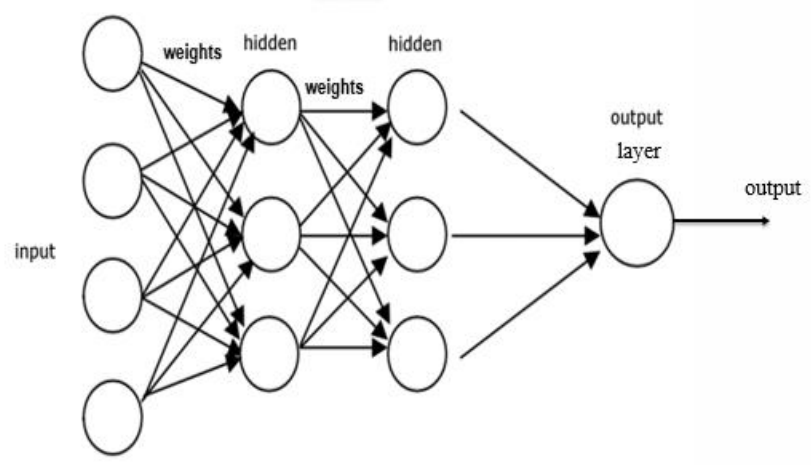

Figure 4 Architecture of RNN with 2 hidden layers 


\section{Sigmoid Function}

The range of logistics function is 0 to 1 and it can be expressed in the equation given below where $\mathrm{S}$ denotes the sigmoid function

$$
S=1 /\left(1+e^{-x}\right)
$$

\section{Tanh Function}

The range of logistics function is -1 to 1 and it can be expressed in the equation given

In RNN, the activations arrive at the hidden layer from both the current input and hidden below where $\mathrm{T}$ denotes the tanh function

$$
T=2 /\left(1+e^{-2 x}\right)-1
$$

\subsubsection{Working of RNN}

Layer activation one step back in time.This hidden state holds the past knowledgeof the network at the given time step. The hidden state is updated at each time step by using the recurrence relation 3 .

$$
h_{t}=f w\left(x_{t}, h_{t-1}\right)
$$

where $h_{t}$ is thecurrent state, $h_{t-1}$ is theprevious state and $x_{t}$ isthe input state.

This generated hidden state is then used to generate a new hidden state and so on. In this case, there are four inputs to be given to the network. The same weight and similar function is applied at each time step.

Formula for applying Activation function (tanh)at time $\mathrm{t}$ is given in equation 4.

$$
h_{t}=\tan h\left(w_{h h} h_{t-1}+w_{x h} x_{t}\right)(4)
$$

where $w_{h h}$ is the weight at recurrent neuron, $w_{x h}$ is the weight at input neuron.

The resulting unit activations are propagated through the hidden to output layer.

Now, once the final current state is calculated after all the time steps then the output state is calculated by using the formula 5 .

$$
y_{t}=w_{h y} h_{t}
$$

Where $\mathrm{y}_{\mathrm{t}}$ is the output, $\mathrm{W}_{\text {hy }}$ is the weight at output layer

Let the predicted output of the network at any time step be $y_{\mathrm{t}}$ and the actual output be $\mathrm{y}_{\mathrm{t}}{ }^{1}$. Then the deviation in model values from actual values (error) is given by:

$$
E_{t}=-y_{t}^{\prime} \log \left(y_{t}\right)
$$

This error value is used in gradient descent algorithm of weight updation.

\subsubsection{Disadvantages of RNN}

Although the basic RNN is effective, it can suffer from a significant problem. For deep networks, the Back-Propagation process can lead to the following issues:

- Vanishing Gradients: This type of problem arises when the change in weight (gradients) become very small.

- Exploding Gradients: This problem occurs when the gradients become too large due to back propagation.

\subsection{Long Short-Term Memory}

LSTM networks are an extension for RNN networks, which basically extends their memory. The units of an LSTM are used as building units for the layers of a RNN, which is then often called an RNN- LSTM network. The LSTM recurrent unit - remember all the past knowledge and forget irrelevant data by introducing different activation function layers called gates 


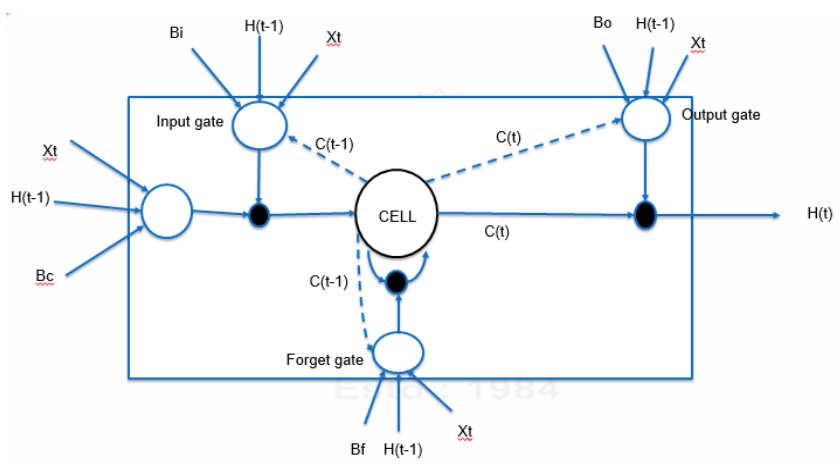

Figure 4 Architecture of RNN-LSTM

$\mathrm{X}_{\mathrm{t}}$ is the current input, $\mathrm{H}_{\mathrm{t}-1}$ is the previous output, $\mathrm{C}_{\mathrm{t}-1}$ is the previous cell state memory, $\mathrm{H}_{\mathrm{t}}$ is the current output, $\mathrm{C}_{\mathrm{t}}$ is the current cell state memory

\subsubsection{Working of RNN-LSTM}

Each LSTM recurrent unit also maintains a vector called the Internal Cell State which describes the information that was chosen to be retained by the previous LSTM recurrent unit. LSTM takes the input of previous current state, previous internal state and current input. The values of four different gates is calculated by using the function given below, where the $\boldsymbol{\sigma}$ (sigmoid) and tanh are the activation functions.

Forget gate $\left(\mathrm{f}_{\mathrm{t}}\right)$ : The information that are not useful no more is removed.

$\mathrm{f}_{\mathrm{t}}=\boldsymbol{\sigma}\left(\mathrm{W}_{\mathrm{xf}} \mathrm{X}_{\mathrm{t}}+\mathrm{W}_{\mathrm{hf}} \mathrm{h}_{\mathrm{t}-1}+\mathrm{W}_{\mathrm{cf}} \mathrm{c}_{\mathrm{t}-1}+\mathrm{b}_{\mathrm{f}}\right)$

Input gate $\left(\mathrm{i}_{\mathrm{t}}\right)$ : Additional information that are useful to the cell state is included

$\mathrm{i}_{\mathrm{t}}=\boldsymbol{\sigma}\left(\mathrm{W}_{\mathrm{xi}} \mathrm{X}_{\mathrm{t}}+\mathrm{W}_{\mathrm{hi}} \mathrm{h}_{\mathrm{t}-1}+\mathrm{W}_{\mathrm{ci}} \mathrm{c}_{\mathrm{t}}+\mathrm{b}_{\mathrm{i}}\right)$

Output gate $\left(\mathrm{o}_{\mathrm{t}}\right)$ : The information that are useful are extracted from the current cell state and given as an output.

$$
\mathrm{O}_{\mathrm{t}}=\boldsymbol{\sigma}\left(\mathrm{W}_{\mathrm{xo}} \mathrm{X}_{\mathrm{t}}+\mathrm{W}_{\mathrm{ho}} \mathrm{h}_{\mathrm{t}-1}+\mathrm{W}_{\mathrm{co}} \mathrm{c}_{\mathrm{t}-1}+\mathrm{b}_{\mathrm{o}}\right)
$$

Current internal cell state $\left(C_{t}\right)$ is calculated by element-wise multiplication of forget gate and the previous internal cell state and then adding with vector of input gate as given in equation 11.

$$
\mathrm{C}_{\mathrm{t}}=\mathrm{f}_{\mathrm{t}} \mathrm{c}_{\mathrm{t}-1}+\mathrm{i}_{\mathrm{t}} \tanh \left(\mathrm{w}_{\mathrm{xc}} \mathrm{X}_{\mathrm{t}}+\mathrm{w}_{\mathrm{hch}} \mathrm{h}_{\mathrm{t}-1}+\mathrm{b}_{\mathrm{c}}\right)
$$

Current hidden state $\left(\mathrm{h}_{\mathrm{t}}\right)$ is calculated by element-wise multiplication of the hyperbolic tangent of the current internal cell state vector and the output gate as given in equation 12 .

$\mathrm{h}_{\mathrm{t}}=\mathrm{O}_{\mathrm{t}} \tanh (\mathrm{ct})$

RNN-LSTM network also generates an output error $E_{t}$ at each time step and this output is used to train the network using gradient descent. If the gradient starts converging towards zero, then the weights of the gates can be adjusted accordingly to bring it closer to 1 .

\subsubsection{Advantages of LSTM-RNN over RNN}

LSTM are a special kind of RNN, capable of learning long-term dependencies and predict the time series data. It deals with the exploding and vanishing gradient problems that can be encountered when training traditional RNN. So LSTM RNN is more efficient than RNN for prediction in Hydroponics system. 


\section{IMPLEMENTATION AND ANALYSIS}

\subsection{Implementation in Traditional Farming}

The electronic circuit for collecting the value of temperature and humidity is implemented in the traditional farming. The values were noted for setting threshold during automation using IoT. The values from sensor is collected using Node MCU ESP8266.This gives the threshold value of temperature and humidity of the implemented region in which the plants can grow effectively.

By measuring so, it is possible to control the motor that is pumping the water to the pipeline to flow only in the time when it is ON through IoT. This controlling process may be done based on the humidity value and temperature. If the temperature is high above the $28^{\circ} \mathrm{c}$ threshold that is set, the sprinkler could be $\mathrm{ON}$ to maintain the high temperature. In the same way, if the humidity of the soil is below the threshold, the water flow through the drip link could be $\mathrm{ON}$ to increase the moisture level of the soil and maintain as the plant requires to make them grow effectively.

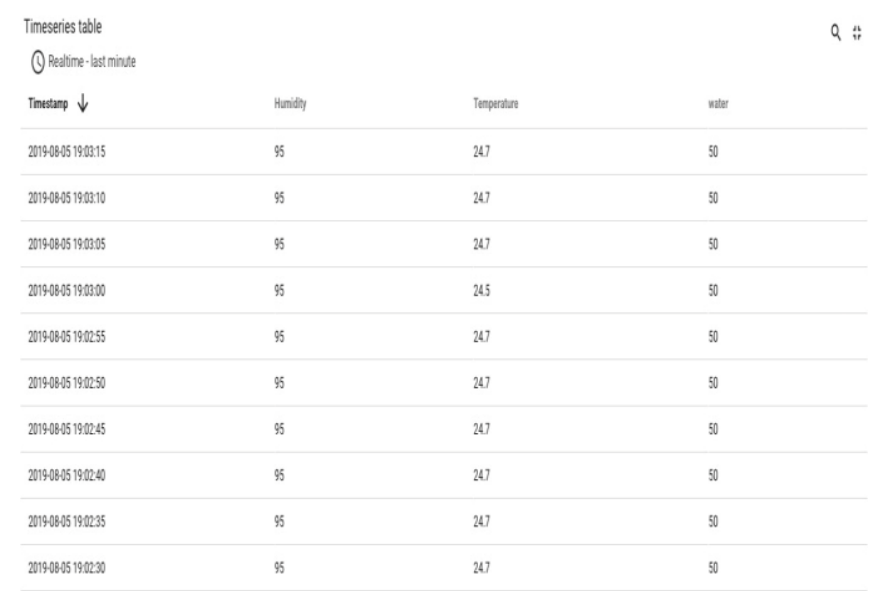

Figure 5 Data collected from traditional framing

\subsection{Implementation in Hydroponics Farming}

Hydroponics technique called Nutrient film technique is implemented. The setup is made automatic by controlling the sprinkler and water flow through pipeline based on the real-time data coming from the sensors (Temperature and Humidity).

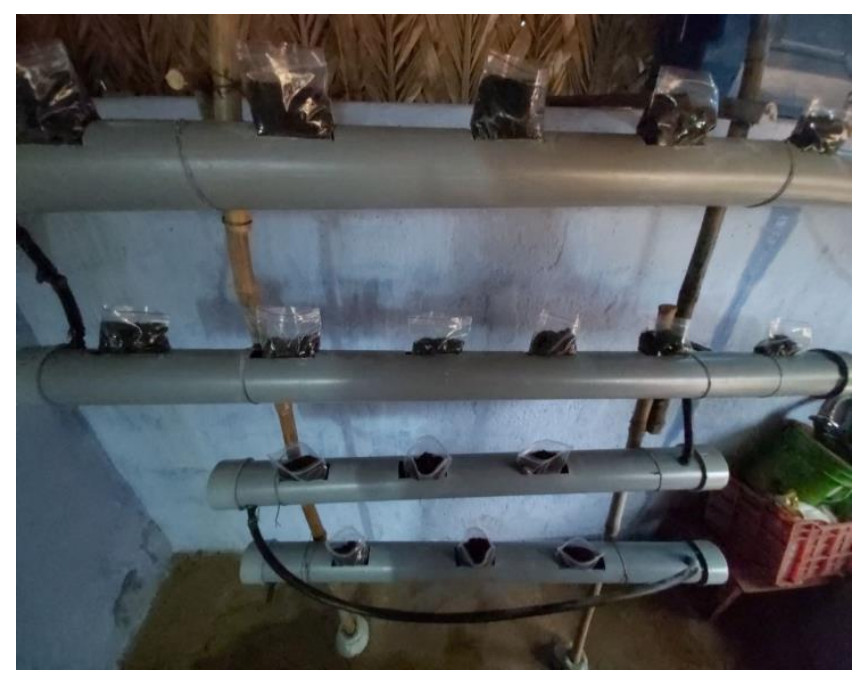

Figure 6 Hydroponics setup 
The materials pipe, end dummy, coconut fiber etc., are used. The holes are drilled according to the distance calculated, 0.75 feet per hole then the mixture of the coconut coir and the coconut dust is packed tightly in the polyethylene cover. This packet is placed into the drilled hole shown in above Figure 6. then the water is feed by turning ON the motor the water is circulated into the setup and all the packets get soaked into the water, all the packets get proper moisture and the seeds of cereals and pulses were sowed into the packets, the setup is moved to get proper sunlight after 3 days seeds started to germinate and the water level and other parameters were monitored by respective sensors continuously and the data were uploaded to the cloud.

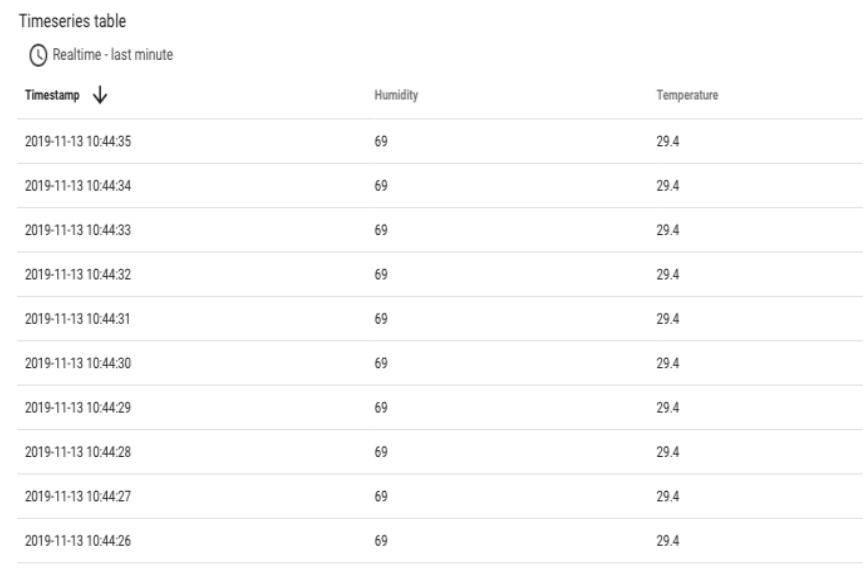

Figure 7 Data collected from Hydroponics framing

\subsection{Prediction Algorithm}

The data collected from the hydroponics are given as input to RNN-LSTM network. The algorithm has been developed in MATLAB.

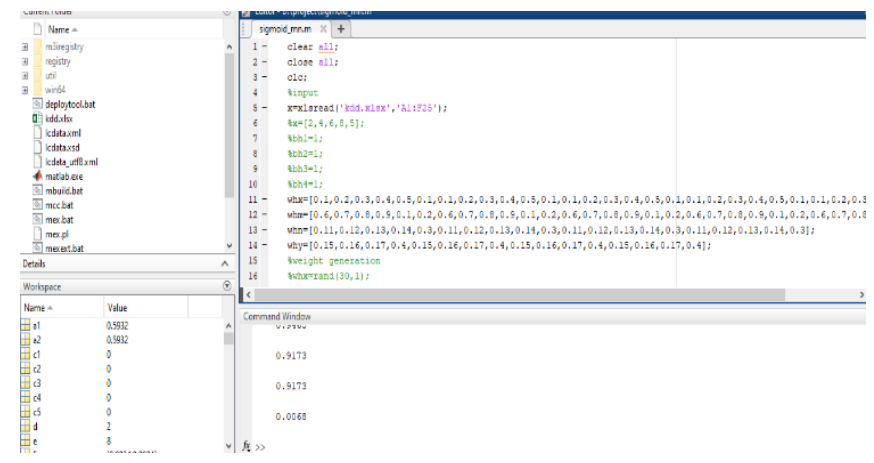

Figure 8 Implementation of RNN-LSTM in MATLAB

The Hydroponics setup's sprinkler could be controlled based on the error value produced as output from the RNN-LSTM algorithm. The sprinkler is in ON state until this error value becomes zero. Results generated in MATLAB is shown in figure 8. Thus the error values from the prediction algorithm can be used to make the hydroponics farming to work based on the temperature and humidity.

\section{CONCLUSION AND FUTURE WORK}

The Hydroponics system is controlled remotely through cloud based on the output from the sensors. Prediction models used in this study were based on a deep learning algorithm, RNNLSTM. Based on the output from these prediction models the system (sprinkler motor, light) 
is controlled through cloud manually. The trained RNN-LSTM could be applied to control nutrient solutions in closed-loop soilless cultures based on prediction of future values of temperature, humidity, and light. This work helps in increasing the productivity and creating an awareness among the farmers to enhance their farming in less cultivable land and water resource areas. There is no need of continuous supervision by the farmers for the cultivation. This work can also implemented in terrace and made as a house hold activity so that everyone can involve in farming and get benefit out of it. Hydroponics farming has made farming very easy. Traditional farming could involve more man-power whereas hydroponics farming does not require any man-power just a vertical farming setup.

Future Work: The error values from the deep neural network are used to automate the entire hydroponics farming.

\section{REFERENCES}

[1] Rajeswari Aravind SS. Inter-American Institute for Cooperation on Agriculture 2009 annual report. 2018.

[2] Sagar J. Dholwani SGMVPP. Introduction of Hydroponic system and it's Methods. Sardar Patel College of Engineering. 2018.

[3] ManavMehra SSRV. IoT based hydroponics system using Deep Neural Networks. SRM Institute of Science and Technology. 2018.

[4] Shreya Tembe SKRA. IoT Based Automated Hydroponics System. International Journal of Scientific \& Engineering Research. 2015.

[5] S. Keerthana KDSSMP. Automating and Analysing Greenhouse Hydroponic Farms using IoT. International Journal for Research in Applied Science \& Engineering Technology (IJRASET). 2018 March.

[6] K. P. Ferentinos LDA. Predictive Neural Network Modelling of $\mathrm{pH}$ and Electrical Conductivity In Deep-trough Hydroponics. American Society of Agricultural Engineers. 2017.

[7] Jitendra Kumara RGAKS. Long Short Term Memory Recurrent Neural Network (LSTMRNN) Based Workload Forecasting Model for Cloud Data centers. National Institute of Technology. 2018.

[8] M. Rakshitha HLSRRT. Automation of Hydroponics System Using Android Application and Ubidots Platform. International Journal of Engineering Research \& Technology (IJERT). 2018.

[9] Chris Jordan G. Aliac EM. IoT Hydroponics Management System. CIT- University. 2018.

[10] IET Digital Library. IET Digital Library. [Online]. [cited 2014 November 27. Available from: http://digital-library.theiet.org/journals/author-guide. 